\title{
Revisiting the Thermodynamic Stability of
}

\section{Indomethacin Polymorphs with}

\section{Low-Frequency Vibrational Spectroscopy and Quantum Mechanical Simulations}

\author{
Michael T. Ruggiero, ${ }^{*, t, \ddagger}$ Joshua J. Sutton, Sara J. Fraser-Miller, Adam J. \\ Zaczek, ${ }^{\S}$ Timothy M. Korter, ${ }^{\S}$ Keith C. Gordon, " and J. Axel Zeitler*,† \\ $\dagger$ Department of Chemical Engineering and Biotechnology, University of Cambridge, New \\ Museums Site, Pembroke Street, Cambridge, CB2 3RA, United Kingdom \\ $\ddagger$ Present Address: Department of Chemistry, University of Vermont, 82 University Place, \\ Burlington, Vermont 05405, United States of America \\ \The Dodd-Walls Centre for Photonic and Quantum Technologies, Department of \\ Chemistry, University of Otago, P.O. Box 56, Dunedin, New Zealand \\ $\S$ Department of Chemistry, Syracuse University, 1-014 Center for Science and Technology, \\ Syracuse, New York, 13244, United States of America \\ E-mail: mtr34@cam.ac.uk; jaz22@cam.ac.uk \\ Phone: +44 (0) 1223 334783; +44 (0) 1223334783
}




\begin{abstract}
The two major polymorphs of the active pharmaceutical ingredient indomethacin were studied using a combination of experimental low-frequency vibrational spectroscopies, theoretical solid-state density functional theory and ab initio molecular dynamics calculations. The results enable a complete spectral assignment of the low-frequency IR and Raman spectra, and yield new insight into the energetic and dynamical factors present within the solids to be understood. Ultimately, these results are used to rationalize the thermodynamic properties of the two crystals, which result in a contradiction to the long-held belief that the $\gamma$-form is the more stable polymorph at ambient conditions due to its predominant abundance. Overall, the study highlights the combined role that molecular conformation, bulk packing arrangement, and intermolecular forces have on the ultimate properties of pharmaceutical crystals, and the need for detailed analyses into all of these effects in order to predict the properties of materials.
\end{abstract}

\title{
Introduction
}

Indomethacin is a non-steroidal anti-inflammatory drug (NSAID) that has been extensively studied over the past several decades, making it a oft-used model system for studying pharmaceutical-related phenomena. ${ }^{1-5}$ Indomethacin is a large and fairly flexible molecule (Figure 1), which enables it to exist in a number of different bulk packing arrangements, exhibiting rich crystalline polymorphism ${ }^{6}$ as well as being easily amorphized. ${ }^{7-10}$ The structural flexibility of indomethacin leads to solids with different energetic, thermal, and overall bulk properties, with one important example being solubility. ${ }^{11-13}$ Given the importance of energetic and thermodynamic parameters on the bulk behavior of active pharmaceutical ingredients, there is a need for an in-depth investigation into these factors, especially for a system as deeply studied as indomethacin.

The crystalline polymorphs of indomethacin have been thoroughly studied over the past several decades by many different methods. There has been particular focus on the two most 


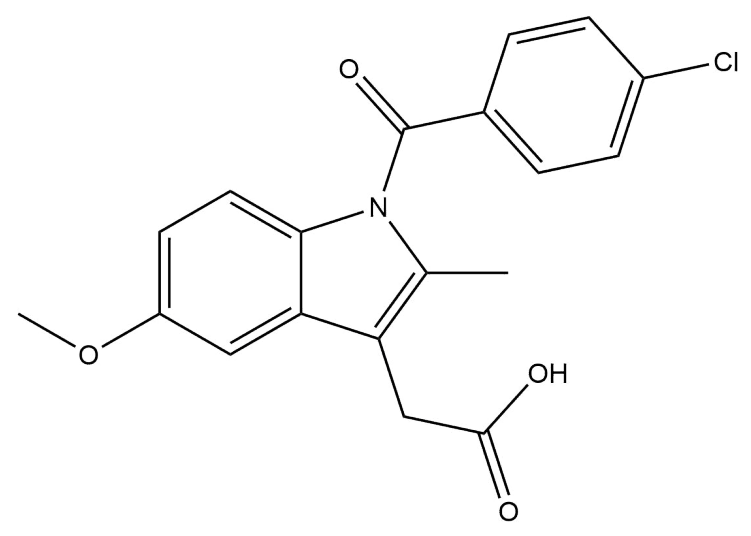

Figure 1: The molecular structure of indomethacin. The molecule has a number of conformational degrees of freedom that enable it to be a fairly flexible system.

common polymorphs, the $\alpha$ - and $\gamma$-forms. ${ }^{6,14-16}$ It is generally accepted that the $\gamma$-form is the more stable solid, with the $\alpha$-polymorph overwhelmingly referred to as 'metastable' in the literature, ${ }^{12,15,17-20}$ despite no reported instances of solid-state phase transformations and all indications that the system is monotropic. The metastable label has been used due to the higher temperatures required to crystallize the $\alpha$-solid. The literature highlights additional peculiarities surrounding the $\alpha$-polymorph, such as a higher crystallographic density (indicating greater stability), ${ }^{17,18}$ yet simultaneously a higher solubility and lower melting point (indicating lower stability), ${ }^{21}$ all compared to the $\gamma$-polymorph. Our findings suggest however that the $\alpha$-polymorph is in fact the more stable polymorph and the term "metastable" is erroneous.

Vibrational spectroscopy is a powerful tool for investigating both the interatomic forces, molecular dynamics, and thermodynamics of molecular solids. ${ }^{22}$ Specifically, low-frequency (3-300 $\left.\mathrm{cm}^{-1}\right)$ vibrational spectroscopy is an especially useful tool, as the associated motions involve entire molecule displacements, for example hindered rotations and molecular torsions, as well as being significant contributors to thermodynamic functions due to the low-energies of the phonon states. ${ }^{23,24}$ The large-amplitude motions ultimately explore large regions of the potential energy hypersurface and by analyzing these vibrations information related to intermolecular interactions, as well as internal conformational energies, can be extracted. ${ }^{25}$ 
However, the complexity of the low-frequency vibrational motions means that advanced computational methods are required in order to interpret and assign the experimental spectra, which are often computationally expensive due to the rigorous criteria necessary to accurately represent the weak forces found in molecular crystals. ${ }^{26-28}$ It is not surprising then that while the low-frequency spectra of the indomethacin polymorphs have been previously reported, ${ }^{29,30}$ no spectral assignment has been performed to date.

The accurate simulation of the low-frequency vibrational features represents a significant challenge to modern computational methods, as the forces involved are exceptionally weak and well-below the traditional error thresholds applied to computational methods. ${ }^{26}$ Therefore, validation of the simulated data is paramount in order to trust the calculated results. The availability of high-quality experimental low-frequency spectroscopic results enables validation of the computed results to be achieved, and allows for additional factors that are difficult to measure experimentally, such as thermodynamic functions, to be reported with confidence. Additionally, the successful modeling of the crystalline structures, low-frequency vibrational transitions, and the corresponding infrared and Raman intensities, provides significant confidence in the utilized theoretical method, as this implies that the potential energy minimum (structure), curvature (vibrational frequency), and atomic charges (IR and Raman intensities) are all accurately reproduced. In this study, the low-frequency terahertz time-domain spectroscopy (THz-TDS) and Raman spectra are reported and assigned using fully periodic density functional theory (DFT) simulations. The results reveal the true nature of the vibrational motions present in these solids, and ultimately enables the reported structural, energetic, and thermodynamic peculiarities to be explained in detail. 


\section{Methods}

\section{Experimental}

\section{Polymorph Preparation}

Commercially available indomethacin ( $\geq 99 \%$, Sigma-Aldrich) is a white crystalline solid comprised of the $\gamma$-polymorph, which was used as-received. The $\alpha$-form was prepared by a modification of previously reported methodologies. ${ }^{1,17}$ The as-received $\gamma$-solid was dissolved in methanol by heating to $60^{\circ} \mathrm{C}$. Upon complete dissolution, the solution was removed from the heat and chilled water was slowly added until a white solid precipitated, which was made up of microcrystalline $\alpha$-indomethacin. The precipitant was filtered and dried over $\mathrm{P}_{4} \mathrm{O}_{10}$, and was used without any further purification.

\section{Terahertz Time-Domain Spectroscopy}

Samples were prepared for THz-TDS measurements by mixing with polyethylene to a $10 \%$ $\mathrm{w} / \mathrm{w}$ concentration, followed by mixing using a mortar and pestle to homogenize the mixture as well as to minimize particle size and reduce scattering effects. ${ }^{31-33}$ The powdered material was then pressed under 2 tons of pressure in a $13 \mathrm{~mm}$ diameter die, yielding a $2 \mathrm{~mm}$ thick freestanding pellet. All THz-TDS spectra were acquired using a commercial TeraPulse 4000 spectrometer (Teraview Ltd.). Cryogenic $(80 \mathrm{~K})$ spectra were collected using a liquid nitrogen cryostat (Janis Research, Wooburn, MA, USA). For each sample, 4900 time-domain waveforms were collected and averaged, and the resultant waveform was Fourier transformed

to yield a terahertz power spectrum with a spectral resolution of $0.94 \mathrm{~cm}^{-1}$. The absorption spectra presented represent the division of a sample power spectra by that of a polyethylene blank spectrum. 


\section{Low-Frequency Raman Spectroscopy}

The low frequency Raman data was collected using a low-frequency Ondax SureBlock THzRaman system with an $830 \mathrm{~nm}$ excitation laser (Ondax, Inc., CA, USA) as previous reported. ${ }^{34,35}$ The SureBlock was fibre optically coupled into an Acton SpectroPro 500i spectrometer (Acton Research, Acton, MA3, USA) and dispersed, via a $1200 \mathrm{~g} / \mathrm{mm}$ grating onto a liquid nitrogen cooled Spec-10:100 B CCD (Princeton Instruments, NJ, USA) operating at $203 \mathrm{~K}$, providing a $2.0 \mathrm{~cm}^{-1}$ spectral resolution. Data was averaged over 5 minutes.

\section{Theoretical}

\section{Solid-State Density Functional Theory}

All simulations were performed using the fully-periodic CRYSTAL17 DFT software package. ${ }^{36}$ The solids were modeled using the Perdew-Burke-Ernzerhof (PBE) density functional, ${ }^{37}$ coupled with the Grimme-D3 dispersion correction including the Becke-Johnson dampening function. ${ }^{38,39}$ The atomic orbitals were represented using the atom-centered split-valence triple- $\zeta$ 6-311G $(2 \mathrm{~d}, 2 \mathrm{p})$ basis set. ${ }^{40}$ Both structures were initially fully optimized (lattice parameters and atomic positions) with no constraints other than the space-group symmetry of the individual solids. Upon complete optimization, vibrational analysis was performed through numerical differentiation of the energy gradient with respect to atomic displacement using a two-point differentiation scheme (program keyword NUMDERIV $=2$ ). ${ }^{41,42}$ Infrared and Raman intensities were determined analytically through the coupled-perturbed Kohn-Sham (CPKS) routine, and the Raman intensities were corrected for experimental conditions (program keyword RAMANEXP). ${ }^{43-45}$ Energy convergence criteria were set to $\Delta E \leq 10^{-8}$ and $10^{-10}$ hartree for the optimization and vibrational calculations, respectively. 


\section{$a b$ initio Molecular Dynamics}

All ab initio molecular dynamics (AIMD) simulations were performed using the open-source and fully-periodic CP2K software package. ${ }^{46,47}$ In order to be comparable to the solidstate DFT simulations, attempts were made to use similar computational parameters wherever possible. The PBE density functional, coupled with the D3 dispersion correction, was used for all simulations. The electronic structure was represented using the DZVPGTH basis set, ${ }^{48}$ combined with the Goedecker-Teter-Hutter (GTH) pseudopotentials. ${ }^{49}$ The isothermal-isobaric (NPT) ensemble was utilized to enable the simulation cell to relax at each temperature, as to accurately take thermal expansion phenomena into account. The

temperature was maintained using a Nosé-Hoover chain thermostat, ${ }^{50-52}$ while the pressure was set to 1 bar using the extended Nosé-Hoover barostat by Martyna et al. ${ }^{53}$ The initial structure was generated by placing four trimer units (12 molecules, 492 atoms total), extracted from the solid-state DFT-optimized $\alpha$-crystal, randomly into a cubic simulation cell such that the density of the system was $10 \%$ lower than the crystalline density. The simulation cell was set to have triclinic Bravais symmetry so that all lattice parameters were free to relax. A 1 fs time-step was used, and the system was equilibrated for 5 ps prior to data collection, which represented a 20 ps trajectory. The convergence criterion was set to $\Delta E \leq 10^{-9}$ hartree.

\section{Results and Discussion}

\section{Structural Analysis}

High-quality structures of both polymorphs of indomethacin have been previously determined experimentally using single-crystal X-ray diffraction measurements at sufficiently low-temperature that they can be compared to the DFT minimizations. ${ }^{17,18}$ The $\alpha$-form crystallizes in the monoclinic $P 2_{1}$ space group, with DFT-optimized lattice parameters of 
$a=5.363 \AA, b=25.454 \AA, c=18.037 \AA$, and $\beta=94.442^{\circ}$, and is shown in Figure 2. There are six molecules in the unit cell $(Z=6)$, with three symmetry unique molecules $\left(Z^{\prime}=3\right)$. The $\gamma$-form crystallizes in the triclinic $P \overline{1}$ space group, with optimized lattice parameters of $a=9.231 \AA, b=9.476 \AA, c=10.772 \AA, \alpha=70.970^{\circ}, \beta=88.138^{\circ}$, and $\gamma=70.217^{\circ}$ (average error of -0.87\%), and is also shown in Figure 2. There are two molecules in the unit cell $(Z=2)$, with one symmetry unique molecule $\left(Z^{\prime}=1\right)$.
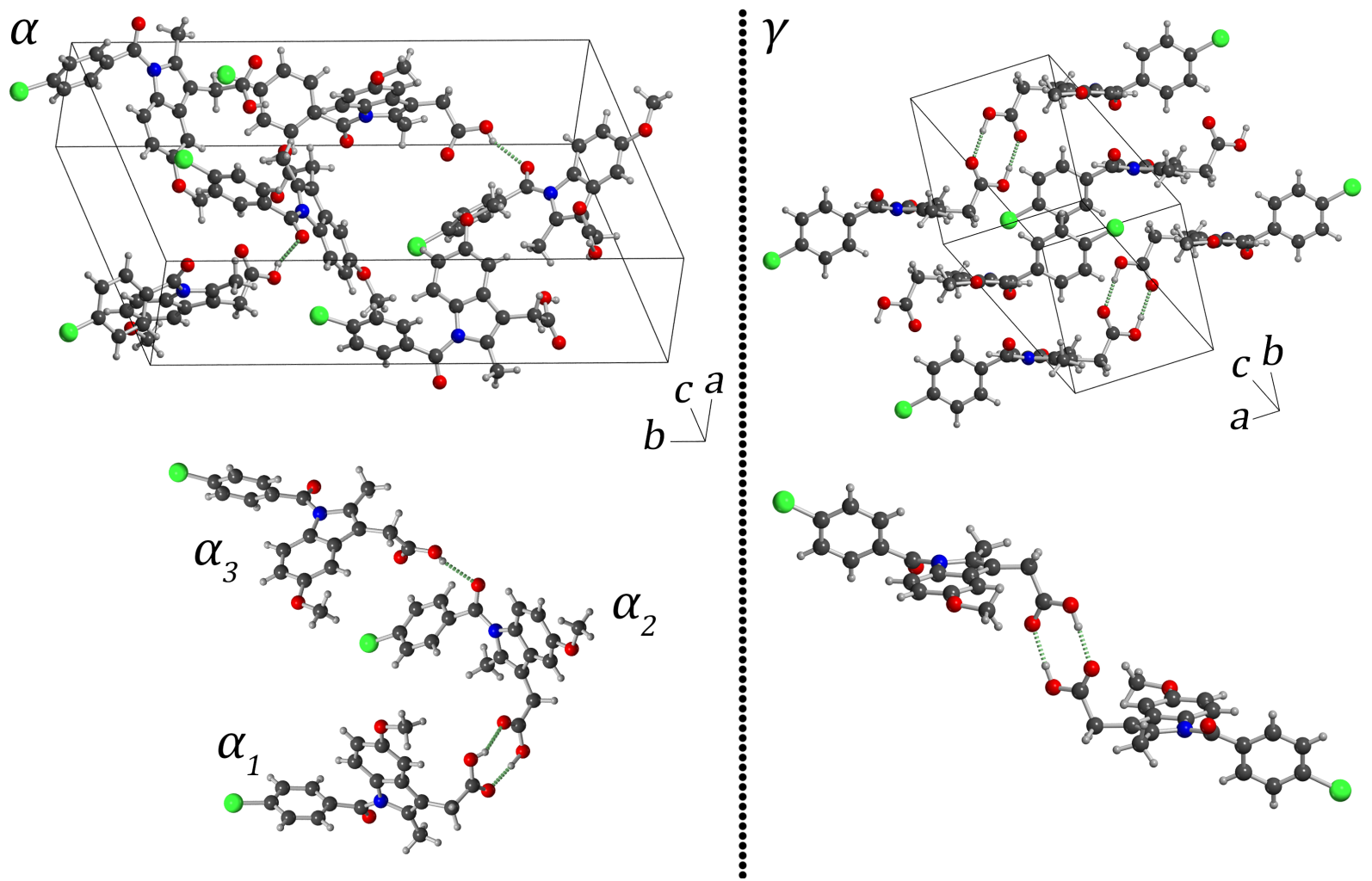

Figure 2: Bulk static structures of the two forms of indomethacin, the $\alpha$-crystal (left) and $\gamma$-crystal (right). Also shown beneath both bulk structures are the main structural units found in both solids, with hydrogen bonds noted by dotted green lines. The three conformations found in the $\alpha$-solid are labelled with subscripts.

The bulk structure of the $\alpha$-polymorph is much more complex then that of the $\gamma$-crystal, due to the three symmetry unique molecular conformations present. The complex molecular conformations enable the formation of a number of intermolecular interactions, including hydrogen bonded carboxylic acid groups between molecules $\alpha_{1}$ and $\alpha_{2}$, a carbonyl-carboxylic acid hydrogen bond between molecules $\alpha_{2}$ and $\alpha_{3}$, and $\pi$-stacking interactions between the 
chlorophenyl rings. The molecules are arranged so that all of the chlorine bonds are unidirectionally oriented along the $b$-axis.

The packing of the $\gamma$-polymorph is much less complex, with two major structural features observed. The first is the presence of two hydrogen bonds between the carboxylic acid groups, forming dimer pairs, while the second is $\pi$-stacking of the chlorophenyl rings (which are arranged orthogonal to the indole ring). The result of these interactions is the bulk formation of planar sheets of the indole-ring system, with the chlorophenyl rings from adjacent sheets interacting via $\pi$-stacking.

The conformations present in the $\alpha$-solid all exhibit significant differences between themselves (and the conformation found in the $\gamma$-form), primarily in the orientation of the chlorophenyl ring with respect to the indole ring, with the $\alpha_{3}$-conformation most similar to the $\gamma$-conformation. Particularly, $\alpha_{2}$ exhibits the most unusual conformation, with the chlorophenyl ring exhibiting an usually short intramolecular cholorophenyl-methyl separation $(2.94 \AA$, Figure 3). This arises due to the nature of the amide dihedral angle, which is rotated by about $180^{\circ}$ compared to the other three conformations. The individual structures are all available for download in the ESI.

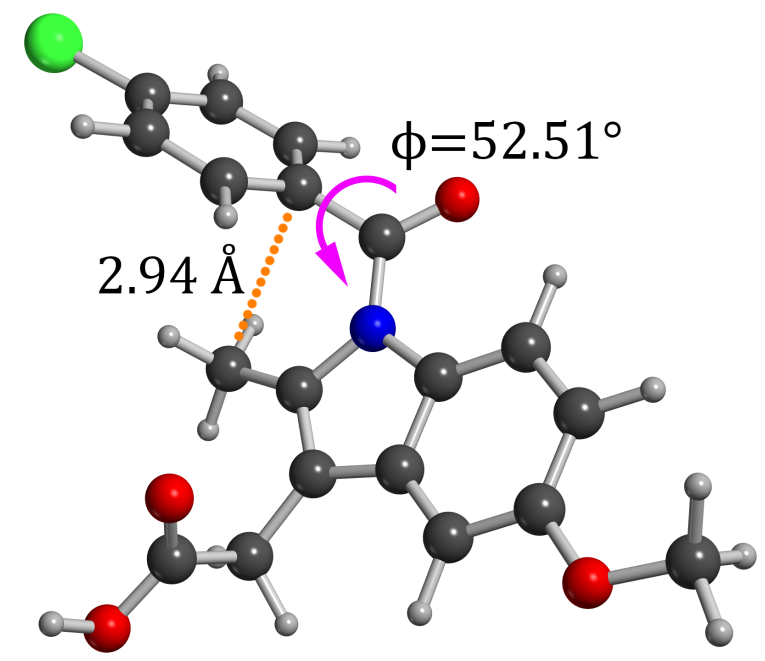

Figure 3: Structure of the $\alpha_{2}$ conformation showing the unusually short cholorophenylmethyl separation (orange dotted line) and the small chlorophenyl-amide torsional angle (purple arrow). 


\section{Low-Frequency Vibrational Results}

The significant structural differences, both in the individual molecular conformations and bulk packing, makes the indomethacin polymorphs suitable materials for investigation using low-frequency vibrational spectroscopies. In order to draw any firm conclusions regarding the energetic and thermodynamic forces governing the solids from the theoretical calculations it is important to have high-quality experimental data to compare to. The low-frequency vibrational spectra provide an excellent dataset to validate the calculations, given that the modes at these frequencies are dominated by weak forces that are represent a significant challenge to reproduce theoretically.

The experimental low-temperature $(80 \mathrm{~K})$ THz-TDS and low-frequency Raman spectroscopy results for the $\alpha$ - and $\gamma$-polymorphs are shown in Figure 4 and Figure 5, respectively. The experimental spectra are clearly different from one another, which is expected due to the contrasting structures of the two solids, and are in good agreement with previously published spectra. ${ }^{29,30}$ While the $\gamma$-form exhibits a number of well-resolved peaks, the $\alpha$-form is characterized by much broader spectral features. This is likely due to the large number of vibrational transitions present in the $\alpha$-crystal, due to the much larger unit cell (and reduced symmetry) yielding a congested low-frequency spectrum, with many individual modes coalescing into broadened spectral features.

In order to analyze and assign the experimental spectra, the vibrational frequencies, and corresponding IR-intensities and Raman-activities, were simulated using the CRYSTAL17 software package. The results, also shown in Figure 4 and Figure 5, represent a good agreement with experiment, confirming the validity of the theoretical model. As expected, the $\alpha$-solid presents a dense low-frequency vibrational density of states, with many vibrational transitions contributing to the bulk spectra. In contrast, the $\gamma$-polymorph exhibits fewer modes, which are well-separated, making individual spectral mode-assignment possible.

Investigation of the vibrational normal modes enables the particular dynamics corresponding to each transition to be obtained, yielding insight into the nature of the interactions 


\section{$\alpha$-Indomethacin}
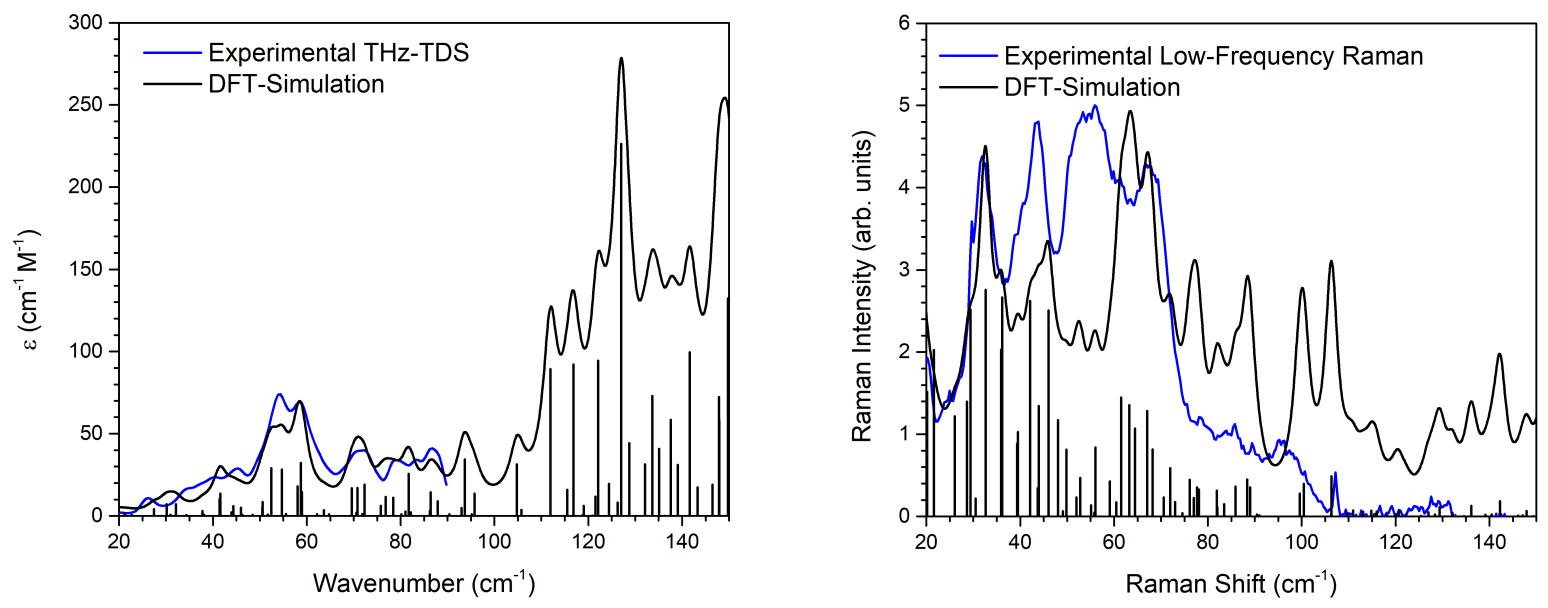

Figure 4: Experimental $80 \mathrm{~K}$ (blue) and DFT-simulated (black) THz-TDS (left) and lowfrequency Raman (right) spectra of $\alpha$-indomethacin. Both figures are plotted beyond the experimental bandwidth of the experimental techniques in order to show the entire lowfrequency vibrational spectra predicted by DFT.
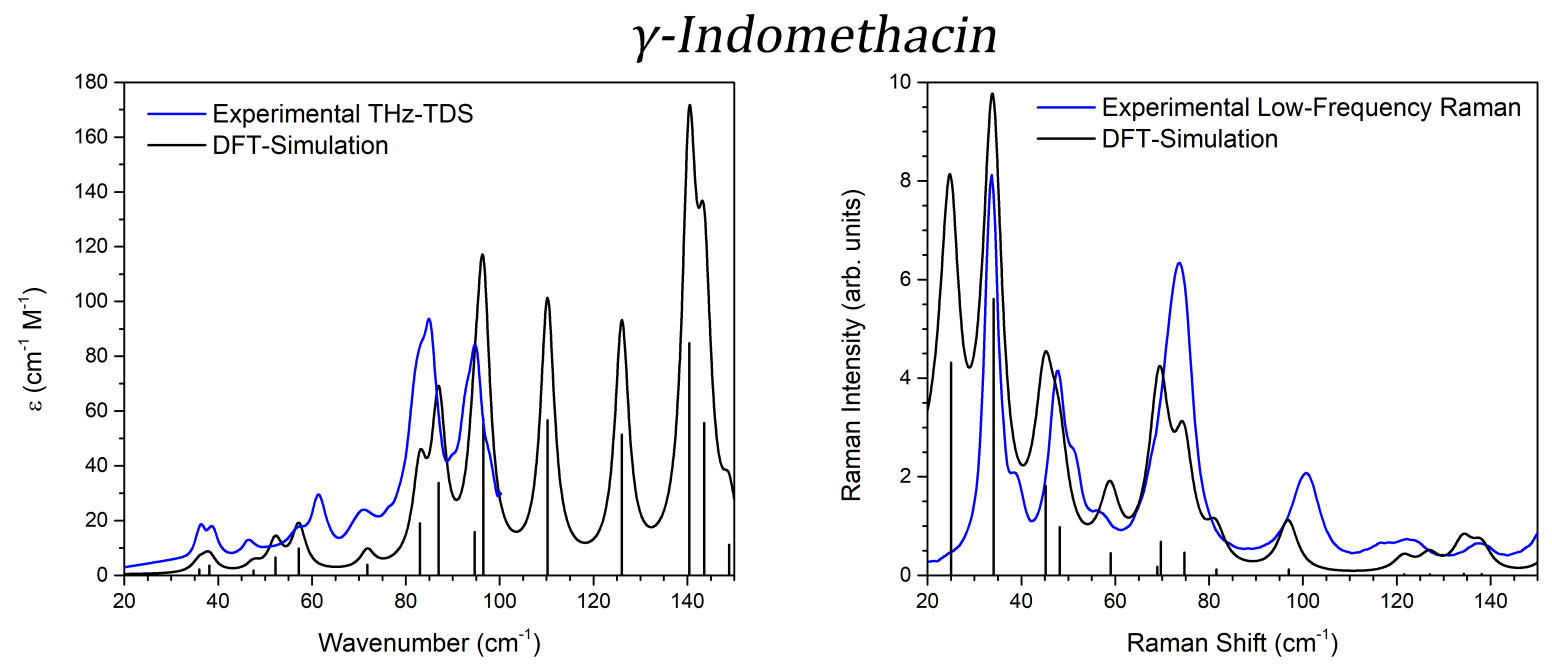

Figure 5: Experimental $80 \mathrm{~K}$ (blue) and DFT-simulated (black) THz-TDS (left) and lowfrequency Raman (right) spectra of $\gamma$-indomethacin. Both figures are plotted beyond the experimental bandwidth of the experimental techniques in order to show the entire lowfrequency vibrational spectra predicted by DFT. 
present in the solid. Overall, the two crystals exhibit very similar vibrational dynamics, with most of the lowest-frequency $\left(<50 \mathrm{~cm}^{-1}\right)$ modes presenting as conformationally-rigid hindered rotational motions, with increasing frequencies leading to more internal torsional-type motions (full mode assignment available in the ESI). In the $\gamma$-solid, all of the vibrations can be considered collective vibrations, given that the crystallographic symmetry of the solid means that adjacent molecules always move with respect to one another. However, in the $\alpha$-crystal, the reduced symmetry results in reduced coupling between molecules in certain modes, and permits the differences in conformation to be inferred based on the frequencies of corresponding vibrational motions. For example, the vibrational modes in the $\alpha$-crystal involve a number of isolated torsional modes that are observed at much lower frequencies than in the $\gamma$-solid, indicating that the internal conformations are weaker. This is especially true for the $\alpha_{2}$ conformation, in that it not only experiences a number of isolated low-frequency torsional modes, but also exhibits vibrations at consistently lower-frequencies compared to the other conformations present within the same solid. For example, the torsion of the $\alpha_{2}$-amide bond, which results in motion of the chlorophenyl-ring with respect to the indole-ring, occurs at a lower frequency than for the other conformations, indicating that this coordinate is weakened with respect to the other conformations. However, the complexity of the vibrational motions makes more detailed analysis challenging.

\section{Energetic and Thermodynamic Analysis}

While inspection of the vibrational motions provide insight into the forces present within these solids, the vibrational modes are too complex, and the crystals too different, for meaningful energetic parameters to be extracted directly. However, the accurate simulation of the vibrational spectra indicates that the structures, energies, forces, and by extension, thermodynamic parameters, are all well described by the theory. It is important to note that all energies, given in units of $\mathrm{kJ} \operatorname{mol}(\text { molecule })^{-1}$, have been corrected for basis set superposition error so that direct comparisons between the two solids can be made, and are provided as 
$\Delta E_{\alpha-\gamma}=E_{\alpha}-E_{\gamma}$.

Considering the total electronic energy alone, the $\alpha$-polymorph is predicted to be more stable by $-4.64 \mathrm{~kJ} \mathrm{~mol}^{-1}$, determined from the fully-periodic simulation including dispersion energy. This is in good agreement with previous reports, ${ }^{11,14}$ which is seemingly contradictory to the generally accepted theory that the $\gamma$-polymorph is the more stable solid. In addition to providing an estimate for the total electronic energy, the DFT calculations allow for the resolution of much finer detail, such as the energy of each conformation by extracting each molecule and simulating the energy in the absence of periodic boundary conditions. The results, shown in Table 1, indicate that two of the three $\alpha$-conformations are significantly higher in energy than the $\gamma$-conformation, while the third was slightly more stable. In the case of the $\alpha_{2}$ conformation, the energy difference is very large $\left(+11.45 \mathrm{~kJ} \mathrm{~mol}^{-1}\right)$, which is intuitive given the observed structural strain (see Figure 3) and vibrational results are all related.

Given that the sum of the conformational energies would result in an electronic energy difference of $+4.21 \mathrm{~kJ} \mathrm{~mol}^{-1}$ on the basis of conformation alone, it is rather surprising that the overall electronic energy for the solid is actually in favor of the $\alpha$-form by $-4.64 \mathrm{~kJ}$ $\mathrm{mol}^{-1}$. This can be explained by calculating the binding energies of the molecules within the solid, determined by taking the difference between the isolated conformational energy and the total bulk electronic energy,

$$
E(\text { binding })=E(\text { unit cell })-\sum_{i=1}^{n} Z_{i} E_{i}
$$

where $n$ is the total number of symmetry unique conformations, $Z_{i}$ is the number of molecules with $i$ conformation within the unit cell, and $E_{i}$ is the energy of the isolated molecule. The calculation of the binding energy for both solids results in a net difference in binding energy of $-8.85 \mathrm{~kJ} \mathrm{~mol}^{-1}$, which is rather large. From this, it is obvious that the bulk packing in the $\alpha$-polymorph is much more favorable than the packing in the $\gamma$-polymorph, even though it comes at the expense of two unfavorable molecular conformations. 
Extending the analysis from pure electronic energies (temperature-independent) to thermodynamic functions (temperature-dependent), the results do not change significantly. In fact, the $\alpha$-polymorph is predicted to be even more stable at ambient conditions than what is predicted by pure electronic energy alone $\left(\Delta G_{\alpha-\gamma}^{298 K}=-5.39 \mathrm{~kJ} \mathrm{~mol}^{-1}\right)$. Furthermore, the $\alpha$-solid is predicted to be the more stable polymorph, across all meaningful temperatures where the sample is solid. The simulations predict that the thermodynamic stability of the polymorphs switch at temperatures above $607 \mathrm{~K}$, which is well above the melting point of both solids. While this result should be considered carefully, as the periodic-DFT framework does not take into account important high-temperature parameters such as vibrational anharmonicity and significant thermal expansion effects, as well as constraining the system to the solid state, the data highlights that while both polymorphs are solids there is no evidence of a solid-state phase transformation (see Supplementary Information). While this indicates that the system is monotropic, which is generally accepted experimentally, it is contradictory to the long-held belief that the $\gamma$-polymorph is the thermodynamically stable solid. It is important to explicitly note that this study is concerned only with solid-state stability, and phenomena such as melting are not captured by the utilized methodology. Nonetheless, the results presented indicate that at all temperatures, where both materials are solids, the $\alpha$-polymorph is more stable.

A number of apparent contradictions are apparent related to the indomethacin polymorphs. For instance, the $\alpha$-polymorph is known to have a higher density, a general indication of the more stable solid, ${ }^{17,18}$ yet it also requires heat to form and has been shown to have a higher solubility than the $\gamma$-form, ${ }^{13}$ which is commonly interpreted to be asociated with lower stability. However, the detailed energetic results provide greater insight into the origins of these effects, and the unstable $\alpha_{2}$ conformation and resulting bulk structure are paramount to rationalizing the physical observations.

The reason why heat is required for the crystallization of the $\alpha$ form can be explained in terms of the highly strained $\alpha_{2}$ conformation that results in a higher thermal energy barrier 
Table 1: Energetic differences between the $\alpha$ - and $\gamma$-indomethacin polymorphs, as well as the difference between an isolated conformation and all of the indomethacin polymorphic conformations. All energies are given in $\mathrm{kJ} \mathrm{mol}^{-1}$

\begin{tabular}{|c|c|c|c|c|c|}
\hline \multicolumn{6}{|c|}{ Bulk Energies } \\
\hline & \multicolumn{2}{|c|}{ Electronic } & & \multicolumn{2}{|c|}{ Thermodynamic $(298 \mathrm{~K})$} \\
\hline & Total & Binding & & Gibbs & Entropy \\
\hline$\Delta_{\alpha-\gamma}$ & -4.64 & -8.85 & & -5.39 & $+3.85 \times 10^{-3}$ \\
\hline \multicolumn{6}{|c|}{ Conformational Energies } \\
\hline & & $\alpha_{1}$ & $\alpha_{2}$ & $\alpha_{3}$ & \\
\hline$\Delta_{\alpha-\gamma}$ & & +2.38 & +11.45 & -1.19 & \\
\hline \multicolumn{6}{|c|}{ Packing-Induced Conformational Strain } \\
\hline$\Delta$ & $\begin{array}{c}\gamma \\
+22.75\end{array}$ & $\begin{array}{c}\alpha_{1} \\
+25.13\end{array}$ & $\begin{array}{c}\alpha_{2} \\
+34.20\end{array}$ & $\begin{array}{c}\alpha_{3} \\
+21.55\end{array}$ & \\
\hline
\end{tabular}

compared to the other conformations. It is therefore not surprising that the formation of this conformation is not observed at low temperatures. However, once sufficient energy is available the benefit of the cumulative lower energy of the $\alpha$-form due to the favorable packing (i.e. the binding energy) dominates, which then drives crystallization. Thus, while the formation of $\alpha$-crystals is thermodynamically favored, there are significant kinetic barriers hindering formation, ultimately resulting in $\gamma$-crystal growth at lower-temperatures.

The solubility advantage of the $\alpha$-polymorph is two-fold. The surface structure of $\alpha$ crystals exhibits dangling bonding sites, which are not available in the $\gamma$-form. ${ }^{17}$ This leads to an increased likelihood of surface molecules interacting with solvent molecules, which faciliitates the dissolution process. In addition, the higher strain associated with the $\alpha_{2}$ molecule leads to a significant energetic advantage for that particular molecule to become solvated (and adopt a more favorable structure), which further contributes to the increased solubility.

In order to further investigate this hypothesis, AIMD simulations were performed on a simulation cell containing 4 indomethacin trimers (extracted from the $\alpha$-bulk) that were initially randomly placed within a cubic simulation cell. Simulations were performed within the NPT ensemble at four temperatures $(200,300,400$, and $500 \mathrm{~K})$. After a 5 ps equilibra- 


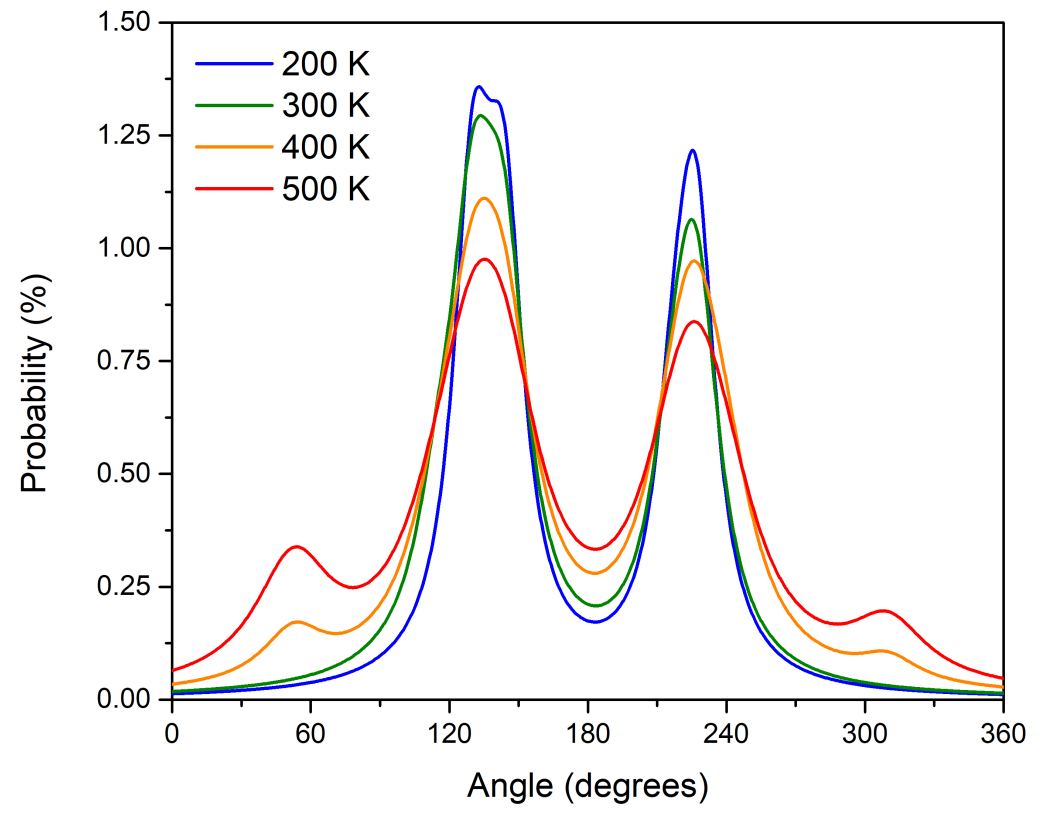

Figure 6: The AIMD-simulated dihedral angle distribution function for indomethacin at four temperatures, 200,300,400, and $500 \mathrm{~K}$ (blue, green, orange, and red, respectively). Dihedral angles $\approx 60^{\circ}$ correspond to the $\alpha_{2}$ conformation, while angles $\approx 125^{\circ}$ correspond to the $\gamma$ and $\alpha_{3}$ conformations and $\approx 230^{\circ}$ the $\alpha_{1}$ conformation. The peak occurring $\approx 310^{\circ}$ does not correspond to any conformation found in the crystalline solids. The data have been normalized such that the integral of the each function was equal to one. 
tion, 20 ps trajectories were simulated and the dihedral distribution function for the amidechlorophenyl torsion (see Figure 3) was determined. Figure 6 shows that at temperatures below $400 \mathrm{~K}$, the molecules tend to adopt conformations corresponding to either the $\alpha_{3} / \gamma$ or the $\alpha_{1}$ conformations, with little-to-no probability of forming the $\alpha_{3}$ conformation. Above $400 \mathrm{~K}$ the simulations indicate that there is some significant probability of forming the $\alpha_{3}$ conformation, as well as forming a fourth conformation that does not correspond to any of the conformations found in either polymorph. The occurrence of the additional structural conformations highlights the required thermal energy required to overcome potential energy barriers corresponding to conformational rearrangement. In this case, the appearance of the two new peaks in the dihedral-angle distribution function correspond to the onset of nearly free structural rearrangement around the chlorophenyl group. Such phenomena have been observed before in a molecular glass former, where this type of transition corresponds to the 'glass-transition' of the material. ${ }^{54}$ While these simulations are not an exact representation of the crystallization conditions used (i.e. a lack of solvent molecules), they provide significant insight into the thermal energies required to form the unfavorable structure that is necessary for crystallization of the $\alpha$-polymorph, and ultimately agree well with the temperatures required to form the $\alpha$-polymorph experimentally.

\section{Conclusions}

Overall, the combination of experimental terahertz time-domain spectroscopy, low-frequency Raman spectroscopy, theoretical solid-state density functional theory, and ab initio molecular dynamics simulations enables a complete picture of the structural, dynamical, energetic, and thermodynamic parameters of the two major polymorphs of indomethacin to be obtained. The results indicate that contrary to what is accepted, it is the $\alpha$-polymorph that is more thermodynamically stable to a significant degree, with the important caveat being within the solid state. The origins of the apparent instability arise from the conformations of the 
particular molecules present within the solids, with the $\alpha$-crystal containing a particularily unstable molecular conformation the presents a significant kinetic barrier to its formation at ambient conditions. Through the use of ab initio molecular dynamics simulations, the temperatures required for the formation of the unfavorable conformation are significantly higher than ambient conditions, helping to confirm the kinetic barrier picture. Ultimately, the results showcase the complex interplay of forces found within molecular crystals, and how subtle atomic-level properties can have a significant outcome on bulk properties.

\section{Acknowledgments}

MTR and JAZ thank the UK Engineering and Physical Sciences Research Council (EPSRC) for funding (EP/N022769/1) and HEC Materials Chemistry Consortium for access to the ARCHER UK national supercomputer (EP/L000202). KCG and JEB acknowledge the support of the Dodd-Walls Centre for Photonic and Quantum Technologies. TMK and AJZ thank Syracuse University for their continued support.

\section{Supporting Information}

Mode assignment for the low-frequency region for both polymorphs. This material is available free of charge via the Internet at http://pubs.acs.org.

\section{References}

(1) Strachan, C. J.; Rades, T.; Gordon, K. C. A Theoretical And Spectroscopic Study of Gamma-crystalline And Amorphous Indometacin. J. Pharm. Pharmacol 2007, 59, $261-269$.

(2) Taylor, L. S.; Zografi, G. Spectroscopic Characterization of Interactions Between Pvp 
And Indomethacin in Amorphous Molecular Dispersions. Pharm. Res. 1997, 14, 16911698.

(3) Varughese, P.; Li, J.; Wang, W.; Winstead, D. Supercritical Antisolvent Processing of $\gamma$-indomethacin: Effects of Solvent, Concentration, Pressure And Temperature On Sas Processed Indomethacin. Powder Technol. 2010, 201, 64-69.

(4) Savolainen, M.; Heinz, A.; Strachan, C.; Gordon, K. C.; Yliruusi, J.; Rades, T.; Sandler, N. Screening For Differences in the Amorphous State of Indomethacin Using Multivariate Visualization. Eur. J. Pharm. Sci. 2007, 30, 113-123.

(5) Semjonov, K.; Kogermann, K.; Laidmäe, I.; Antikainen, O.; Strachan, C. J.; Ehlers, H.; Yliruusi, J.; Heinämäki, J. The Formation And Physical Stability of Two-phase Solid Dispersion Systems of Indomethacin in Supercooled Molten Mixtures with Different Matrix Formers. Eur. J. Pharm. Sci. 2017, 97, 237-246.

(6) Surwase, S. A.; Boetker, J. P.; Saville, D.; Boyd, B. J.; Gordon, K. C.; Peltonen, L.; Strachan, C. J. Indomethacin: New Polymorphs of an Old Drug. Mol. Pharmaceutics 2013, 10, 4472-4480.

(7) Yoshioka, M.; Hancock, B. C.; Zografi, G. Crystallization of Indomethacin From the Amorphous State Below And Above Its Glass Transition Temperature. J. Pharm. Sci. 1994, 83, 1700-1705.

(8) Andronis, V.; Zografi, G. The Molecular Mobility of Supercooled Amorphous Indomethacin As a Function of Temperature And Relative Humidity. Pharm. Res. 1998, $15,835-842$.

(9) Andronis, V.; Yoshioka, M.; Zografi, G. Effects of Sorbed Water On the Crystallization of Indomethacin From the Amorphous State. J. Pharm. Sci. 1997, 86, 346-351. 
(10) Lu, Q.; Zografi, G. Phase Behavior of Binary And Ternary Amorphous Mixtures Containing Indomethacin, Citric Acid, And Pvp. Pharm. Res. 1998, 15, 1202-1206.

(11) Aubrey-medendorp, C.; Swadley, M. J.; Li, T. The Polymorphism of Indomethacin: an Analysis By Density Functional Theory Calculations. Pharm. Res. 2008, 25, 953-959.

(12) Legendre, B.; Feutelais, Y. Polymorphic And Thermodynamic Study of Indomethacin. J. Therm. Anal. Cal. 2004, 76, 255-264.

(13) Pudipeddi, M.; Serajuddin, A. T. M. Trends in Solubility of Polymorphs. J. Pharm. Sci. 2005, 94, 929-939.

(14) Aceves Hernandez, J. M.; Nicolás Vázquez, I.; Aceves, F. J.; Hinojosa Torres, J.; Paz, M.; Castaño, V. M. Indomethacin Polymorphs: Experimental And Conformational Analysis. J. Pharm. Sci. 2009, 98, 2448-2463.

(15) Carpentier, L.; Decressain, R.; Desprez, S.; Descamps, M. Dynamics of the amorphous and crystalline alpha-, gamma-phases of indomethacin. J. Phys. Chem. B 2006, 110, $457-464$.

(16) Andronis, V.; Zografi, G. Crystal Nucleation And Growth of Indomethacin Polymorphs From the Amorphous State. J. Non-Cryst. Solids 2000, 271, 236-248.

(17) Chen, X.; Morris, K. R.; Griesser, U. J.; Byrn, S. R.; Stowell, J. G. Reactivity Differences of Indomethacin Solid Forms with Ammonia Gas. J. Am. Chem. Soc. 2002, 124, $15012-15019$.

(18) Cox, P. J.; Manson, P. L. $\gamma$-indomethacin At 120 K. Acta Crystallogr., Sect. E: Struct. Rep. Online 2003, 59, o986-o988.

(19) Desprez, S.; Descamps, M. Transformations of Glassy Indomethacin Induced By Ballmilling. J. Non-Cryst. Solids 2006, 352, 4480-4485. 
(20) Cox, J. R.; Ferris, L. A.; Thalladi, V. R. Selective Growth of a Stable Drug Polymorph By Suppressing the Nucleation of Corresponding Metastable Polymorphs. Angew. Chem. Int. Ed. 2007, 46, 4333-4336.

(21) Hancock, B. C.; Parks, M. What Is the True Solubility Advantage For Amorphous Pharmaceuticals? Pharm. Res. 2000, 17, 397-404.

(22) Beran, G. J. O. Modeling Polymorphic Molecular Crystals with Electronic Structure Theory. Chem. Rev. 2016, 116, 5567-5613.

(23) Kittel, C. Introduction to Solid State Physics; A Modern Account which Stresses Elementary Physical Models of Solids; Wiley, 1959.

(24) Einstein, A. Die Plancksche Theorie Der Strahlung Und Die Theorie Der Spezifischen Wärme. Ann. Phys. 1907, 327, 180-190.

(25) Parrott, E. P. J.; Zeitler, J. A. Terahertz Time-domain And Low-frequency Raman Spectroscopy of Organic Materials. Appl. Spectrosc. 2015, 69, 1-25.

(26) Ruggiero, M. T.; Zeitler, J. A.; Erba, A. Intermolecular Anharmonicity in Molecular Crystals: Interplay Between Experimental Low-frequency Dynamics And Quantum Quasi-harmonic Simulations of Solid Purine. Chem. Commun. 2017, 53, 3781-3784.

(27) Delaney, S. P.; Pan, D.; Yin, S. X.; Smith, T. M. Evaluating the Roles of Conformational Strain And Cohesive Binding in Crystalline Polymorphs of Aripiprazole. Cryst. Growth Des. 2013, 13, 2943-2952.

(28) Delaney, S. P.; Pan, D.; Galella, M.; Yin, S. X.; Korter, T. M. Understanding the Origins of Conformational Disorder in the Crystalline Polymorphs of Irbesartan. Cryst. Growth Des. 2012, 12, 5017-5024.

(29) Shibata, T.; Mori, T.; Kojima, S. Low-frequency Vibrational Properties of Crystalline 
And Glassy Indomethacin Probed By Terahertz Time-domain Spectroscopy And Lowfrequency Raman Scattering. Spectrochim. Acta, Part A 2015, 150, 207-211.

(30) Hedoux, A.; Paccou, L.; Guinet, Y.; Willart, J.-f.; Descamps, M. What Is the True Solubility Advantage For Amorphous Pharmaceuticals? Eur. J. Pharm. Sci. 2009, 38, $156-164$.

(31) Shen, Y. C.; Taday, P. F.; Pepper, M. Elimination of Scattering Effects in Spectral Measurement of Granulated Materials Using Terahertz Pulsed Spectroscopy. Appl. Phys. Lett. 2008, 92, 051103.

(32) Strachan, C. J.; Rades, T.; Newnham, D. A.; Gordon, K. C.; Pepper, M.; Taday, P. F. Using Terahertz Pulsed Spectroscopy to Study Crystallinity of Pharmaceutical Materials. Chem. Phys. Lett. 2004, 390, 20-24.

(33) Bardon, T.; May, R. K.; Taday, P. F.; Strlič, M. Influence of Particle Size On Optical Constants From Pellets Measured With Terahertz Pulsed Spectroscopy. IEEE Trans. Terahertz Sci. Technol. 6, 408-413.

(34) Mah, P. T.; Fraser, S. J.; Reish, M. E.; Rades, T.; Gordon, K. C.; Strachan, C. J. Use of Low-frequency Raman Spectroscopy And Chemometrics For the Quantification of Crystallinity in Amorphous Griseofulvin Tablets. Vib. Spectrosc. 2015, 77, 10-16.

(35) Lipiäinen, T.; Fraser-Miller, S. J.; Gordon, K. C.; Strachan, C. J. Direct Comparison of Low- And Mid-frequency Raman Spectroscopy For Quantitative Solid-state Pharmaceutical Analysis. J. Pharm. Biomed. Anal. 2018, 149, 343-350.

(36) Dovesi, R.; Erba, A.; Orlando, R.; Zicovich Wilson, C. M.; Civalleri, B.; Maschio, L.; Rérat, M.; Casassa, S.; Baima, J.; Salustro, S. et al. Quantum-mechanical Condensed Matter Simulations with Crystal. WIREs Comput Mol Sci 2018, 110, e1360. 
(37) Perdew, J.; Burke, K.; Ernzerhof, M. Generalized Gradient Approximation Made Simple. Phys. Rev. Lett. 1996, 77, 3865-3868.

(38) Grimme, S.; Antony, J.; Ehrlich, S.; Krieg, H. A Consistent And Accurate ab initio Parametrization of Density Functional Dispersion Correction (DFT-D) For the 94 Elements H-Pu. J. Chem. Phys. 2010, 132, 154104.

(39) Grimme, S.; Ehrlich, S.; Goerigk, L. Effect of the Damping Function in Dispersion Corrected Density Functional Theory. J. Comput. Chem. 2011, 32, 1456-1465.

(40) Krishnan, R.; Binkley, J. S.; Seeger, R.; Pople, J. A. Self-consistent Molecular Orbital Methods. Xx. A Basis Set For Correlated Wave Functions. J. Chem. Phys. 1980, 72, $650-654$.

(41) Pascale, F.; Zicovich-Wilson, C. M.; Gejo, F. L.; Civalleri, B.; Orlando, R.; Dovesi, R. The Calculation of the Vibrational Frequencies of Crystalline Compounds And Its Implementation in the Crystal Code. J. Comput. Chem. 2004, 25, 888-897.

(42) Zicovich-Wilson, C. M.; Pascale, F.; Roetti, C.; Saunders, V. R.; Orlando, R.; Dovesi, R. Calculation of the Vibration Frequencies of Alpha-quartz: the Effect of Hamiltonian And Basis Set. J. Comput. Chem. 2004, 25, 1873-1881.

(43) Maschio, L.; Kirtman, B.; Rérat, M.; Orlando, R.; Dovesi, R. Ab initio Analytical Raman Intensities For Periodic Systems Through a Coupled Perturbed Hartree-fock/kohnsham Method in an Atomic Orbital Basis. I. Theory. J. Chem. Phys. 2013, 139, 164101.

(44) Maschio, L.; Kirtman, B.; Rérat, M.; Orlando, R.; Dovesi, R. Ab initio Analytical Raman Intensities For Periodic Systems Through a Coupled Perturbed Hartree-fock/kohnsham Method in an Atomic Orbital Basis. Ii. Validation And Comparison with Experiments. J. Chem. Phys. 2013, 139, 164102. 
(45) Maschio, L.; Kirtman, B.; Rérat, M.; Orlando, R.; Dovesi, R. Comment On "Ab Initioanalytical Infrared Intensities For Periodic Systems Through a Coupled Perturbed Hartree-Fock/Kohn-Sham Method" [J. Chem. Phys. 137, 204113 (2012)]. J. Chem. Phys. 2013, 139, 167101.

(46) Hutter, J.; Iannuzzi, M.; Schiffmann, F.; Vandevondele, J. Cp2k: Atomistic Simulations of Condensed Matter Systems. WIREs Comput Mol Sci 2013, 4, 15-25.

(47) Vandevondele, J.; Krack, M.; Mohamed, F.; Parrinello, M.; Chassaing, T.; Hutter, J. Quickstep: Fast And Accurate Density Functional Calculations Using a Mixed Gaussian And Plane Waves Approach. Comput. Phys. Commun. 2005, 167, 103-128.

(48) Vandevondele, J.; Hutter, J. Gaussian Basis Sets For Accurate Calculations On Molecular Systems in Gas And Condensed Phases. J. Chem. Phys. 2007, 127, 114105.

(49) Goedecker, S.; Teter, M. Separable Dual-space Gaussian Pseudopotentials. Phys. Rev. $B$ 1996, 54, 1703-1710.

(50) Nosé, S. A Molecular Dynamics Method For Simulations in the Canonical Ensemble. Mol. Phys. 2002, 100, 191-198.

(51) Nosé, S. A Unified Formulation of the Constant Temperature Molecular Dynamics Methods. J. Chem. Phys. 1998, 81, 511-519.

(52) Martyna, G. J.; Klein, M. L.; Tuckerman, M. Nosé-hoover Chains: The Canonical Ensemble Via Continuous Dynamics. J. Chem. Phys. 1992, 97, 2635.

(53) Martyna, G. J.; Tobias, D. J.; Klein, M. L. Constant Pressure Molecular Dynamics Algorithms. J. Chem. Phys. 1994, 101, 4177-4189.

(54) Ruggiero, M. T.; Krynski, M.; Kissi, E. O.; Sibik, J.; Markl, D; Tan, N. Y.; Arslanov, D.; Van Der Zande, W.; Redlich, B.; Korter, T. M.; Grohganz, H.; Löbmann, K.; Rades, T.; 
Elliott, S. R.; Zeitler, J. A. The Significance of the Amorphous Potential Energy Landscape for Dictating Glassy Dynamics and Driving Solid-State Crystallisation. Phys. Chem. Chem. Phys. 2017, 19, 30039-30047 


\section{For Table of Contents Use Only}

Revisiting the Thermodynamic Stability of Indomethacin Polymorphs with Low-Frequency Vibrational Spectroscopy and Quantum Mechanical Simulations

Michael T. Ruggiero, Joshua J. Sutton, Sara J. Fraser-Miller, Adam J. Zaczek, Timothy M. Korter, Keith C. Gordon, and J. Axel Zeitler
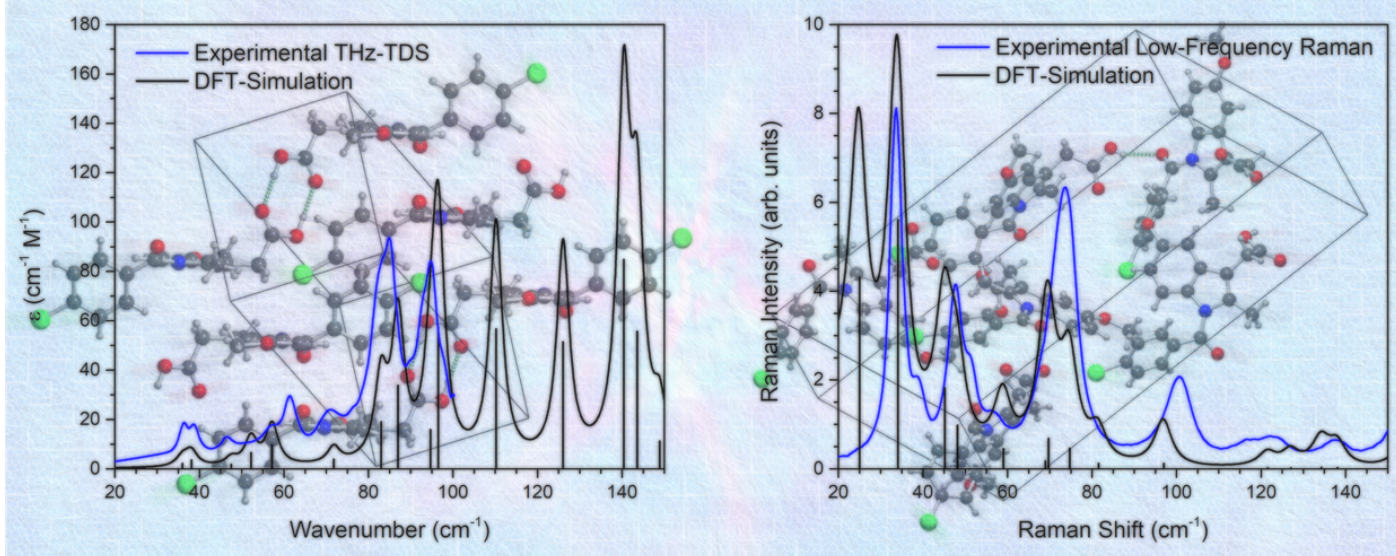

TOC Synopsis: A combination of experimental terahertz time-domain spectroscopy, lowfrequency Raman spectroscopy, and quantum mechanical simulations were used to interpret the thermodynamic stabilities of two indomethacin polymorphs. 